\title{
Reducing Energy Consumption and Operating Costs in C-RAN Based on DWDM/SCM-PON-RoF
}

\author{
W. H. Al-Zubaedi, S. R. Aldaeabool, M. F. Abbod, H. S. Al-Raweshidy \\ Brunel University London, Department of Electrical and Computer Engineering \\ email:wesam.al-zubaedi@brunel.ac.uk
}

\begin{abstract}
This paper proposed to reduce power consumption and cost of operation in the BBU pool by switch off number of BBUs that work at low traffic load. Two algorithms have been proposed, one to convert the operation processing of switched off BBUs to the remaining ones depending on their valuable capacities. The other one is to control the electronic Smart Switch (SS) in order to exchange the traffic load between the Optical Transmitters (OTXs). Mathematical representation model of power consumption for $\mathrm{C}-\mathrm{RAN}$ has been modified. The C-RAN applied by using the DWDM/SCM-PON-RoF architecture to decrease the cost effectiveness. The results in system model validation showed a reduction in the BBUs utilization of $84 \%$ and $83 \%$ in residential and office area respectively. Furthermore, OTXs operation has been reduced by $86 \%$ during the 24 hours compared with the conventional RAN network.
\end{abstract}

\section{INTRODUCTION}

Over the past few years, the demand for the traffic data in cellular network has been steadily increased by developing smart devices and a huge spread internet with new applications. The 5 th Generation $(5 \mathrm{G})$ of the mobile network has been proposed to face this issue. The most significant asset for $5 \mathrm{G}$ to provide data requirements is Radio Access Network (RAN) [1]. It is expected the RAN that represent by microwave back-haul link cannot meet the demands of increasing data rate. Therefore, High capacity Radio over Fiber (RoF) has been proposed to replace the microwave back-haul to provide these needs [2]. Cloud-RAN (C-RAN) architecture innovated as a one of the $5 \mathrm{G}$ solutions to control and simplify the management of the mobile network. The RoF is a technology of integration of optical fiber and wireless access network, where the optical fiber used as a medium to connect the Base Band Units (BBUs) to Remote Radio Heads (RRHs) in the C-RAN network $[3,4]$. Wavelength Division Multiplexing (WDM) and Sub-CarrierMultiplexing (SCM) techniques are the most effective in RoF technology to enhance the communication link between the BBU and RRHs. They can achieve high data rate per wavelength channel and provide high quality service and low power consumption. Passive Optical Networks (PONs) technology is easily computable with the WDM/SCM-RoF to achieve a cost effective system [5]. Dense wavelength division multiplexing (DWDM) technology has been proposed to increase the transmission capacity of lines within multiplexes more than 132 channels with frequency spacing between them less than $200 \mathrm{GHz}$ [6]. The traffic data change for the geographical area and over time during the 24 hour, i.e. a huge amount of energy will waste in the area that users have moved. To achieve an optimal solution to save energy in 5G-RAN that needs to optimize the number of active/sleep a RRHs/BBUs with respect to the traffic demand. The C-RAN gives high reliability to dynamically share of BBUs in BBU pool where can run multiple of RRHs by one BBU special at low data rate demand. Therefore, many studies focused on the cooperation of the BBUs and switching system in Central Office (CO) to save energy. The [7] proposed a technique to reduce energy in C-RAN architecture based on BBUs workload consolidation by reducing the number of BBUs servers used. The technique is switch off idle BBUs to reduce the energy consumption in system. BBU-RRH switching schemes for centralized RAN consider another scenario of cooperative BBU-RRH [8]. In this work the BBUs can be reduced by $79 \%$ and $77 \%$ for the offices and residential area respectively compared with the conventional RAN network. the [9] proposed a scheme to associate BBU and RRH based on graph method to reduce the power consumption in the BBU pool. Simulation results show that the algorithm minimizes power consumption about to 20. M. Khan and his group presented the algorithm to save energy in C-RAN architecture by optimizes the BBUs resource allocation based on active sleep mode technology to about $70 \%$ [10].

This paper proposed a cost effective scheme for C-RAN by changing the connection between BBUs dynamically with respect to the data traffic demand of RRHs. The number of the operating BBUs has been reduced to provide energy saving by sleeping certain BBUs that experiencing low traffic load in particular times of the day. The new approach considers two algorithms. The first one is to toggle the task of sleeping BBUs to the light loaded units according to their available capacity. The task of the second algorithm enables the (SS) to exchange the traffic load between the (OTXs). The proposed model takes into the account the power consumed by optical fibre. To this end, the DWDM/SCM-PON-RoF network has been applied to design C-RAN.

This paper is organized as follows. Section II shows the proposed architecture and calculation of the power consumption model. The calculations of traffic load estimations are illustrated in section III. Section IV shows the algorithms for switching BBUs and OTXs. Section V presents energy saving calculations while experimental set-up and discussion result clarified in section VI. Finally, section VII concludes the work. 


\section{System AND POWER MOdEL}

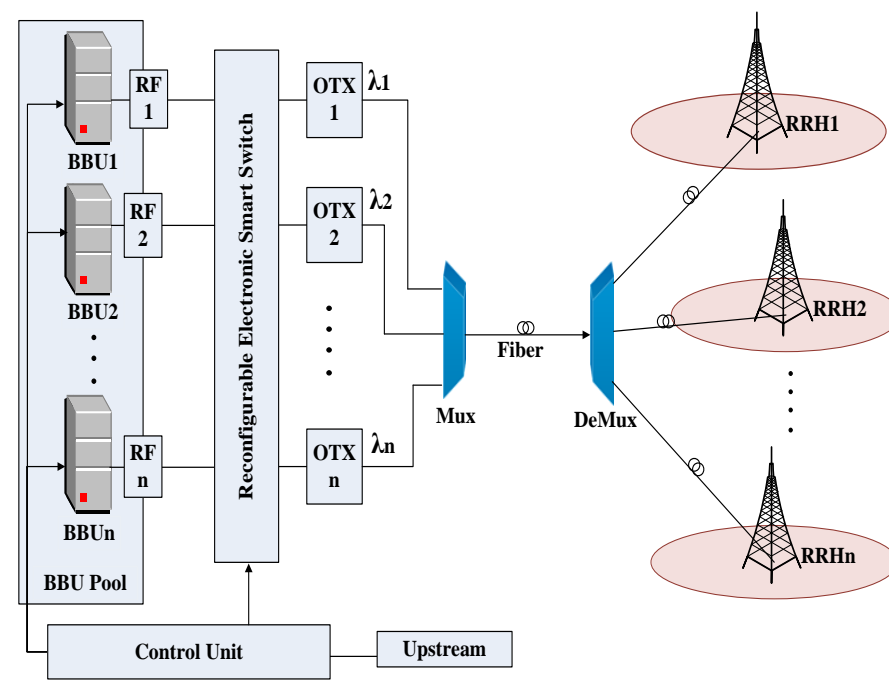

Fig. 1. Proposed architecture of saving energy and cost of operation in CRAN based on Electronic Smart Switch

Figure 1 depicts the proposed system which based on CloudRoF (C-RoF) to simplify the RRH by moving the DAC/ADC to $\mathrm{BBU}$ that used Analogue RoF (A-RoF)for the front-haul network and has benefits that multi technology can coexist in the same optical fibre like in the air while, when use the Digital-RoF (D-RoF) the RRH must be equipped by an $\mathrm{RF}$ path that varies from operator to another operator [4]. It composed of the $\mathrm{CO}$ that consists of number of BBUs, $B B U_{i}=1,2, \ldots, I$, Radio Frequency $R F_{j}=1,2, \ldots, J$ that represented the front-end unit of BBUs, Optical Line Terminal (OLT) that represent the optical part at $\mathrm{CO}$, where, the Optical Transmitters (i.e. Line Card which consumes power about 57 volte) $O T X_{m}=1,2, \ldots, M$ which convert the electrical signal to optical signal, $\mathrm{CU}$ witch control all BBUs within BBU Pools, SS and Multiplexer (Mux). The BBUs connect between them by optical fibre interface and each BBU is controlled number of RRHs, $R R H_{k}=1,2, \ldots, K$. A RoF technology is used as a front-haul medium to connect the BBUs to RRHs by using optical fibre. On cell site the optical signals separates by DeMultiblex (DeMux) then connect to the cell that contain on RRHs that convert the optical signal to RF by $(\mathrm{O} / \mathrm{E})$ converter. A Mux and DeMux used to reduce the cost by enabling to transmit multi wavelengths in one channel instead of multi separated channels. The electronic SS design to make reconfiguration of the system as one to one, one to many, many to many and many to one to help the system to save energy, Such as, one BBU can serve many RRHs.

Many of previous studies introduced the power consumption model for the C-RAN architecture [10-12]. A modified power consumption model of the C-RAN with the addition of optical power calculation will be represent in this section. Optical power has a significant effect especially when it used in a huge optical network $[13,14]$. The power consumption in the proposed system will calculate according to the active and sleep mode of operation for C-RAN architecture. From figure 1 the power consumed in the BBU Pool is [10]:

$$
P_{B B U \text { Pool }}= \begin{cases}\sum_{i}^{n} P_{B B U i}+P_{O} & \text { if } 0<P_{\text {out }} \leq P_{\max } \\ P_{\text {sleep }} & \text { if } P_{\text {out }}<0\end{cases}
$$

Where $\sum_{i}^{n} P_{B B U i}$ is the sum of power consumption of all by all the active BBUs in BBU pool and $P_{\text {out }}$ is the output power from the BBUs, $P_{O}$ is another power consumed in BBU such as cooling and environmental control systems, lights, AC/DC and DC/AC conversion losses.

$$
\begin{gathered}
P_{O}=P_{\text {cooling }}+P_{\text {lighing }}+P_{\text {monitoring }}+P_{\text {back-haul }} \\
P_{B B U i}=N_{T R X} \frac{P_{B B}}{\left(1-\sigma_{D C}\right)\left(1-\sigma_{P S}\right)}
\end{gathered}
$$

where, $P_{B B}$ is the power consumption of Base-Band (BB) in one transceiver TRX, the parameters $\sigma_{D C}$ and $\sigma_{P S}$ stand for the loss of efficiency in DC/DC power supply, and AC/DC power supply respectively [11]. The power consumption in optical part front-haul can represent as shown in equations below [15] with use of the WDM.

$P_{\text {front-haul }}=P_{O T X}+10 \log N-$ Insertion losses $+P_{F i b e r}$

And if the WDM is not used the equation (4) will be changed as in equation (5)

$$
P_{\text {front-haul }}=\sum_{m=1}^{M} P_{O T X m}+\sum_{d=1}^{D} P_{\text {Fiber }_{d}}
$$

Where, $P_{O T X}$ denotes the power consumption of the active optical transmitter of the BBU, $N$ number of the active optical transmitter in the BBU pool, the Insertion losses denoted the insertion losses in WDM, and $P_{\text {Fiber }}$ represent the power consumption in active optical fiber, where the Fiber $_{d}=1,2, \ldots, D$.

$$
P_{\text {Fiber }}=P_{\text {in }} e^{(-\alpha L)}
$$

Where, the $\alpha$ is the attenuation coefficient of the optical fibre, $L$ is the fibre length. The power consumption in optical fiber considers very small value therefore, this work ignored the optical fiber power consumption. The optical power consumption at the cell site represent an Optical Network Unit (ONU):

$$
\begin{aligned}
& P_{O N U s}=\sum_{l=1}^{L} P_{l} \\
& P_{l}=P_{a, l}+P_{s, l}
\end{aligned}
$$

Where: $O N U_{l}=1,2, \ldots, L$, the $P_{l}$ represent the power consumed by the ONU (or the transport link $l$ ) in the active mode and sleep mode that represented by $P_{a, l}$ and $P_{s, l}$ respectively. To this end, the power in Optical Network $(\mathrm{ON})$ can be written as in (9).

$$
P_{O N}=P_{\text {front-haul }}+P_{\text {ONUs }}
$$


the power consumed by the RRH is [11].

$$
P_{R R H_{k}}=\frac{\frac{P_{o u t}}{\eta_{A P}\left(1-\sigma_{f e e d}\right)}+P_{R F}}{\left(1-\sigma_{D C}\right)\left(1-\sigma_{P S}\right)}
$$

$\eta_{A P}$ power amplifier efficiency, $\sigma_{f e e d}$ as the feeder loss factor. $P_{R F}$ radio frequency $(\mathrm{RF})$ power amplifier and $P_{\text {out }}$ is the transmit power of RRH to users that depending on the variation of the load from (minimum to maximum). Now, the modification of the power consumption formula in C-RAN can be defined the equation (11):

$$
P_{C-R A N}=P_{B B U \text { Pool }}+P_{O N}+\sum_{k=1}^{K} P_{R R H_{k}}
$$

\section{ESTIMATION OF TRAFFIC LOAD}

The BBUs continuously monitor required load for their RRHs to satisfy this requirements. The percentage of estimation required load can be expressed as:

$$
P_{i}(t)=\left[\frac{n_{i}(t)}{N(k)}\right] \times 100 \%
$$

$n_{i}(t)$ is the amount of actual load required for RRH at the measurement interval time $t, N(k)$ is a total capacity of load of $R R H_{k}$ in term of of PRB, that is managed by one BBU. The $P_{i}(t)$ based on the two load threshold condition $T h_{h i g h}$ and $T h_{\text {low }}$ the BBU is either designated as high load, low load or medium load depending on the load required of its RRH as a shown in table I.

TABLE I

STATUSE OF BBU LOAD

\begin{tabular}{|c|c|}
\hline BBU status & Traffic load condition \\
\hline low load & $P_{i}(t)<T h_{\text {low }}$ \\
\hline medium load & $T h_{\text {low }} \leq P_{i}(t) \leq T h_{\text {high }}$ \\
\hline high load & $P_{i}(t)>T h_{\text {high }}$ \\
\hline
\end{tabular}

The Load Ratio (LR) for the negotiating between the BBUs to make change connection between them. The LR can be defined according to the following equations.

$$
\begin{gathered}
L R=1-\frac{P_{i}(t)}{B B U_{L}+P_{i}(t)}<1, \text { for low load } \\
L R=\frac{B B U_{L}}{B B U_{L}-P_{i}(t)}>1, \text { for medium load }
\end{gathered}
$$

$B B U_{L}$ is a total amount of occupied load resources that can processed in one BBU.

\section{Switching Algorithms of BBUs And OTXs}

The CU gets the information about the whole system simultaneously. Therefore, the CU can determine the load required for each cluster by the equations (12-14) that hold the algorithm of switching BBUs and OTXs to determine the operating condition. This work proposed two assumptions for algorithm 1 , the first one is creating a group (T) for BBUs that need to low traffic demand to make change connection between them. In order to avoid BBUs instability due to rapid load fluctuations and reduce the handover between the BBUs. This strategy is very useful if applied between two areas, one high load and other low load in a specific time and change this statues in other time such as residential area and office area. The second one is creating group group $(\mathrm{H})$ for BBUs that need medium traffic demand to make change connection between them. On the other hand, the SS can save energy by

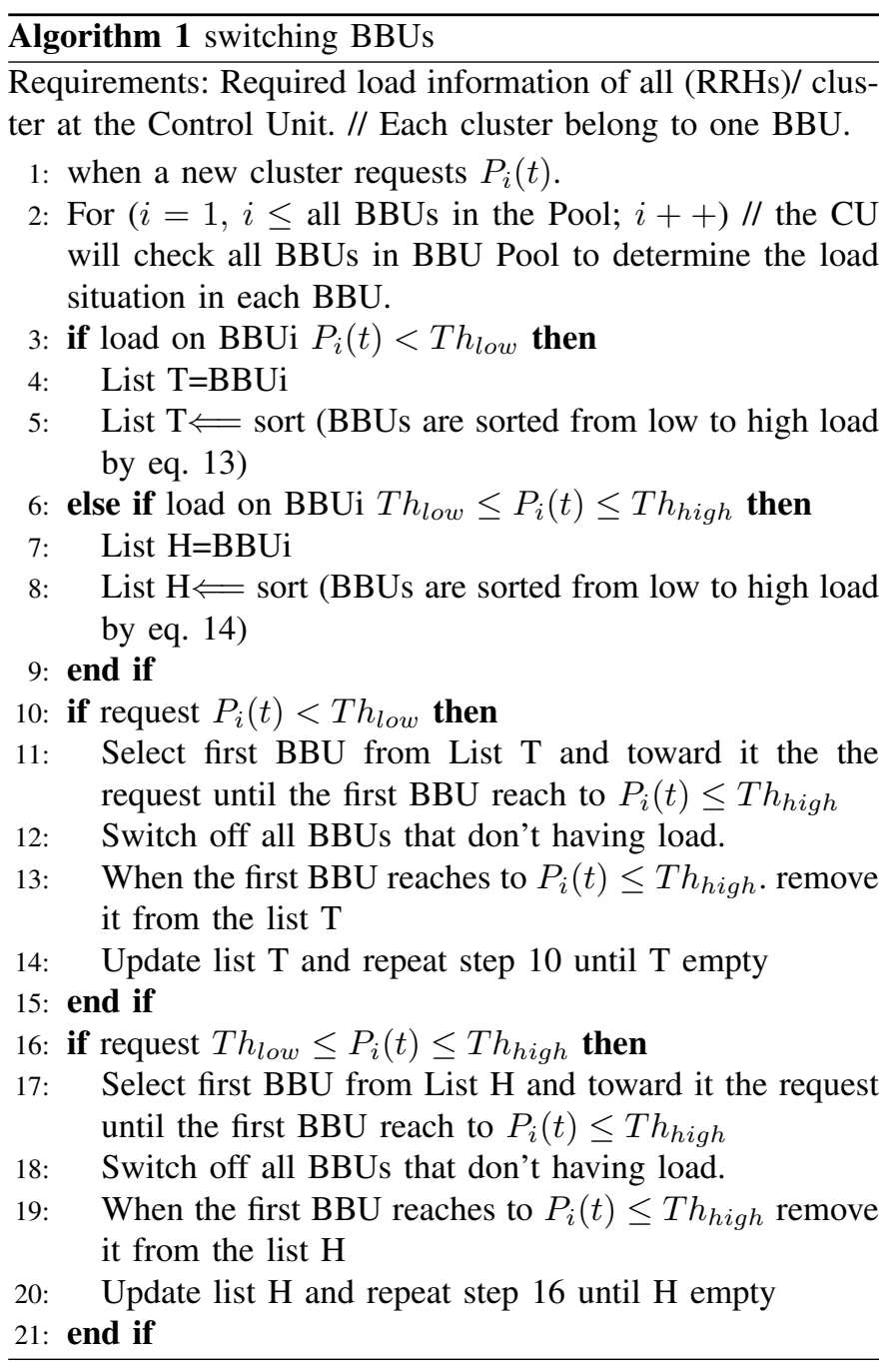

reducing the operation of OTXs. When the RFs inter to the SS, the SS can save energy by rout the RFs that have low and medium load to one OTX if applicable to reduce the number of OTXs operation. This process will show by algorithms below.

\section{EnERgy SAVing CAlculation}

The energy saving and operating costs of conventional RAN and C-RAN network have been studied by the proposed network architecture as shown in figure 1 and simulation condition listed in table II. Energy saving calculation depends 


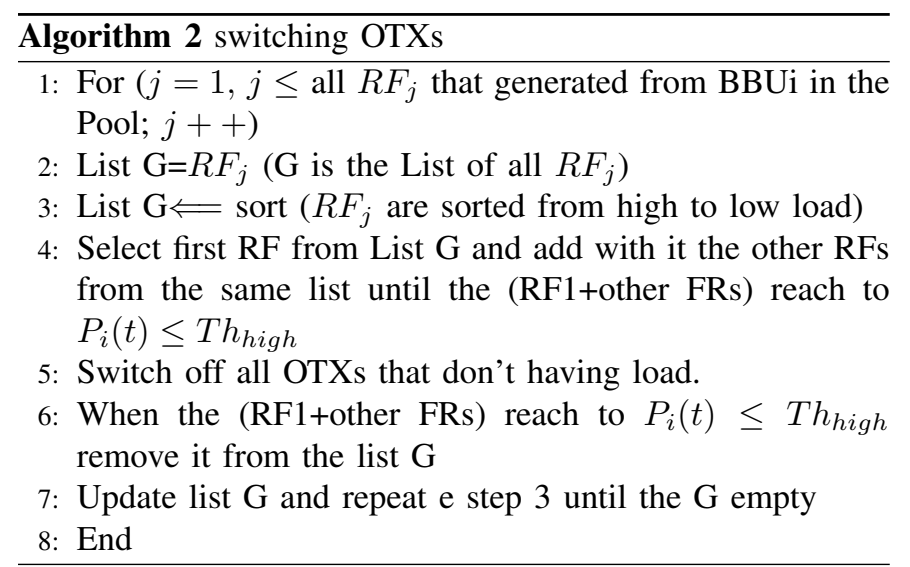

TABLE II

SIMULATION CONDITION

\begin{tabular}{|c|c|}
\hline Parameters & Value \\
\hline Number of operates & 1 \\
\hline Frequencies band & $20 \mathrm{GHz}$ \\
\hline frequency spacing between the BBUs & $200 \mathrm{MHz}$ \\
\hline Number of BBUs & 128 \\
\hline Target time & 24 hours \\
\hline Areas & Residential and Offices area \\
\hline Data rate & $0.4-\mathrm{Gb} / \mathrm{s}$ to $2-\mathrm{Gb} / \mathrm{s}$ \\
\hline$T h_{\text {high }}$ & $90 \%$ \\
\hline$T h_{\text {low }}$ & $20 \%$ \\
\hline
\end{tabular}

on the readings of traffic load for the offices and residential area from reference [10] as shown in figure 2. The

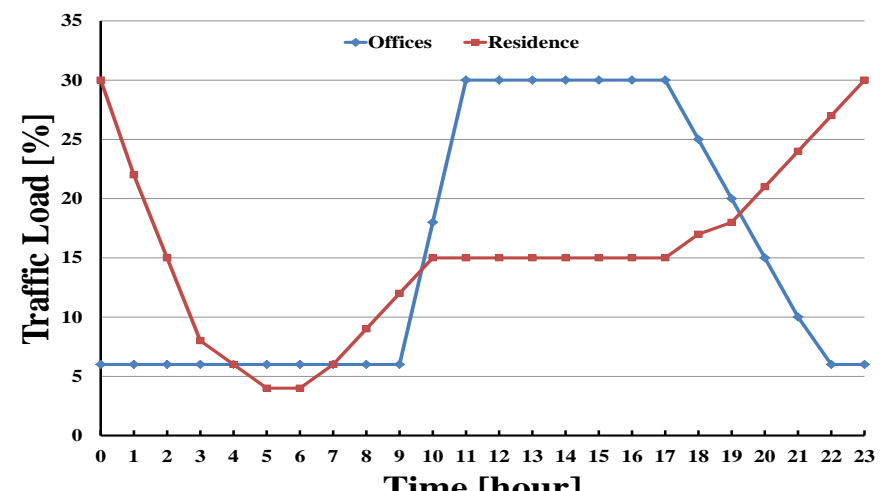

Fig. 2. The percentage of traffic load for offices and residential area during the 24 hours.

figure 3 shows the number of working BBUs for 24 hours of conventional RAN and C-RAN network of offices and residential area respectively. According to our assumption we specified 64 BBUs for each area. By using algorithm 1, CRAN network can reduce the number of working BBUs and energy saving by $84 \%$ and $83 \%$ for the offices and residential area respectively compare with the conventional RAN network while the reference [8] reduced the number of BBUs by $79 \%$ and $77 \%$ for the same areas during 24 hours. Also, the references [10] and [11] reduced the power consumption for the BBUs uses by $70 \%$ and $60 \%$ respectively. The figure

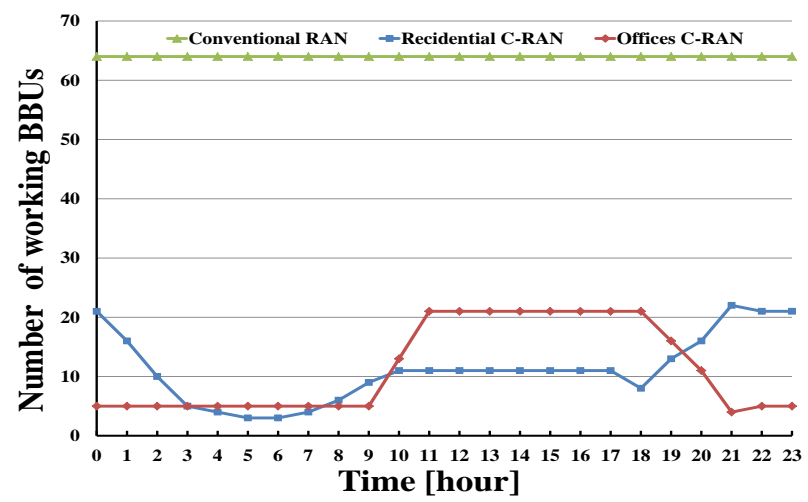

Fig. 3. Number of working BBUs for offices and residential area during 24 hours.

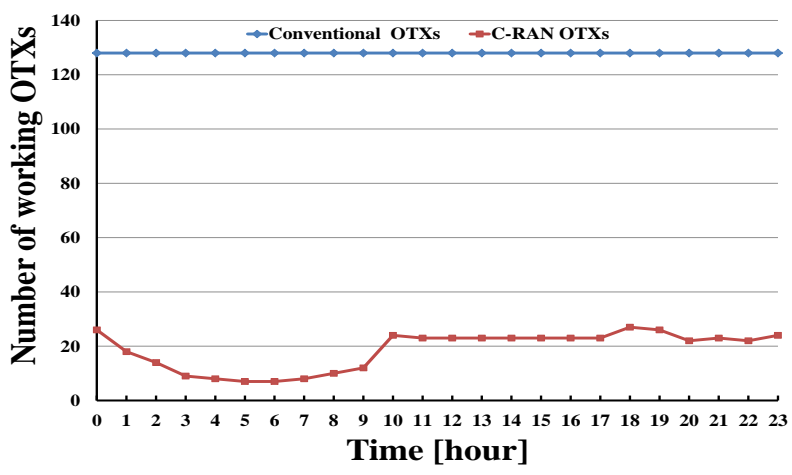

Fig. 4. Number of working OTXs for offices and residential area during 24 hours.

4 show the number of working OTXs during 24 hours for 128 OTXs. According to the algorithm 2 the number of the OTXs reduced by $86 \%$ compare with the conventional RAN network while the reference [9] reduced the average power consumption in OTXs is about by $55 \%$. The references [16] mentioned that power consumption in OLT is fixed while this work proved the opposite by reducing the working OTXs. The two assumptions and algorithms have a powerful effect on the system to reduce the power consumption and operating costs as a shown in previous results.

\section{The Experimental Set-Up AND Discussion RESULT}

The performance of C-RoF technique and the testing of the SS operation are experimental studied in figure 5. The software VPI Transmission Maker was used to simulate the experimental set-up [17]. 4 BBUs was taken to simplify the study performance of the system. The RF generation of each BBU consist of the electrical 64-QAM signal generator an up converted at a given carrier frequency by Local Oscillator (LO) to carry the data for this channel. The given carrier frequencies were $(20 \mathrm{GHz}) \pm 200 \mathrm{MHz}$ for BBUs. The data rate were $2-\mathrm{Gb} / \mathrm{s}$ and $0.4-\mathrm{Gb} / \mathrm{s}$ for high and low traffic demand respec- 


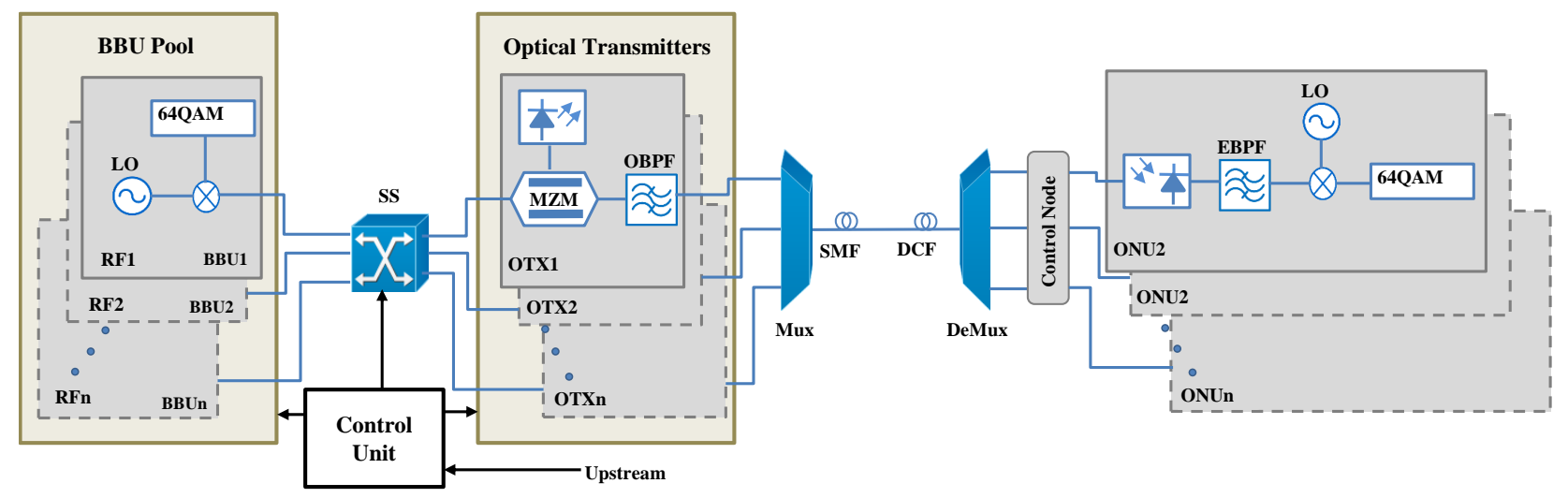

Fig. 5. Experimental Set-Up of proposed system

tively. The OTX consist of Mach-Zehnder Modulator (MZM), Continues Wave (CW) lights from Laser Diodes (LDs) and Optical Band Pass Filter (OPBF). Where, the LDs of BBUs work at the frequencies $(193.25,193.15,193.05$, and 192.95 $\mathrm{THz})$ respectively that correspond to wavelengths $(1552,1553$, 1554 , and $1555 \mathrm{~nm}$ ) at the center frequency is $193.1 \mathrm{THz}$ that correspond to wavelength $1553.5 \mathrm{~nm}$. The system works as DWDM because the frequency spacing between the LDs is 100 GHz. The information modulates via MZM and OPBF used to prevent the interference of other channel frequencies during the transition of the information. Then, four optical channel multiplex by using $4 \times 1$ Mux to transmit the information by Single Mode Fiber (SMF) and Dispersion Compensation Fiber DCF at length 30 and $6 \mathrm{~km}$ respectively. The DCF was used to reduce the dispersion in SMF. On the ONUs side, the DeMux $(1 \mathrm{x} 4)$ used to demultiplex four channels. Connection Node $(\mathrm{CN})$ connects all channels to each photodiode. Four PIN photodiodes (PDs) were used as a receiver to convert the optical signal to an electrical signal. Electrical Band Pass filter (EBPF) is used to separate the signals according their frequencies; where the local oscillator was used for the same frequencies at the transmitter to separate the information from the frequencies to test it experimentally by Rx 64QAM. The $\mathrm{CN}$ used to be suitable for SCM and DWDM system, the CN consist of 4 of ( $1 \times 4$ splitter), each input connect to all RRHs. The SS can rout many RFs (medium and low load to reach to $\left.P_{i}(t) \leq T h_{h i g h}\right)$ to one OTX to carry and modulate by one wavelength as an SCM, then transmit to the clusters and other OTXs will be in a sleep mode. The decodes electrical 64QAM signal that used at the ONUs side to evaluate the optical spectrum and the received constellation of the QAM signal. It is supposed all clusters work at high traffic load, in this case all RFs are routed to their OTXs via the SS to transmit each one by a single wavelength $(1552,1553,1554$, and $1555 \mathrm{~nm})$ to their cluster and system work as DWDM-PON-RoF as a shown in figure 6, that illustrate the optical spectrum over $36 \mathrm{~km}$ fiber length. However, another assumption is all clusters work at low traffic demand, in this case and according to algorithm 2 all RFs routed to OTX1 via SS to transmit all RFs by a

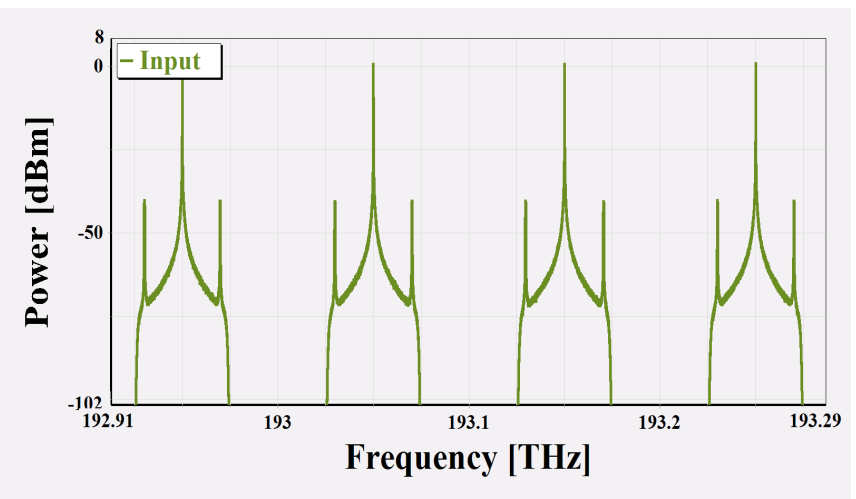

Fig. 6. Optical spectrum for 4 working BBUs with full load.

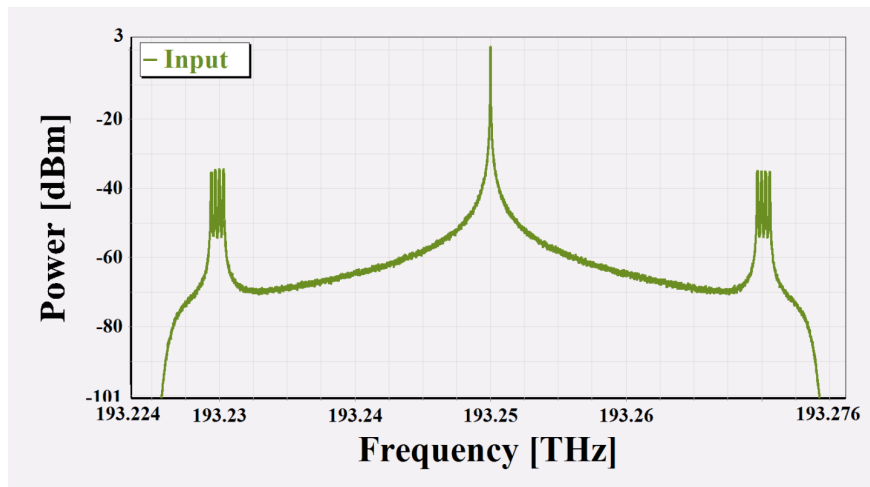

Fig. 7. Optical spectrum for 4 working BBUs with low load that work as a SCM.

single wavelength $(1552 \mathrm{~nm})$ to their cluster and system work as DWDM/SCM-PON-RoF as a shown in figure 7. The figure 8 is zoomed in for the figure 7 to show 4 frequencies carried by one wavelength as an SCM. The system has good response for the constellation diagram at full load traffic demand as a shown in Figure 9. 


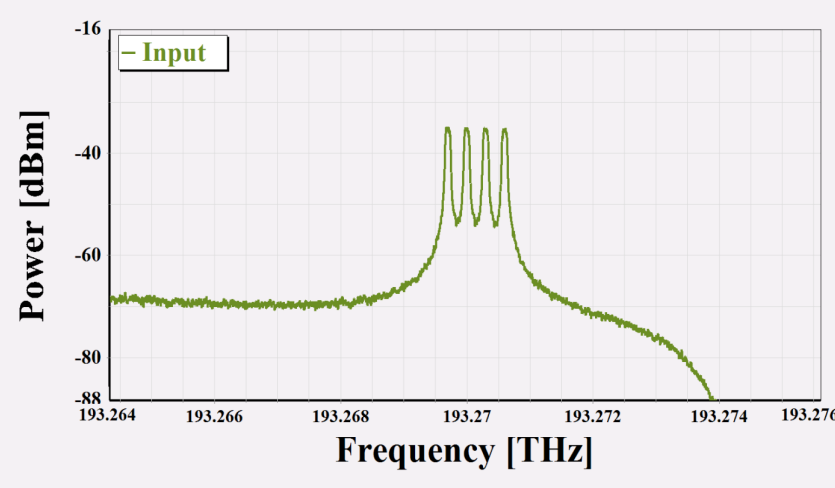

Fig. 8. Optical spectrum for 4 working BBUs with low load after zoom in for figure 7 .

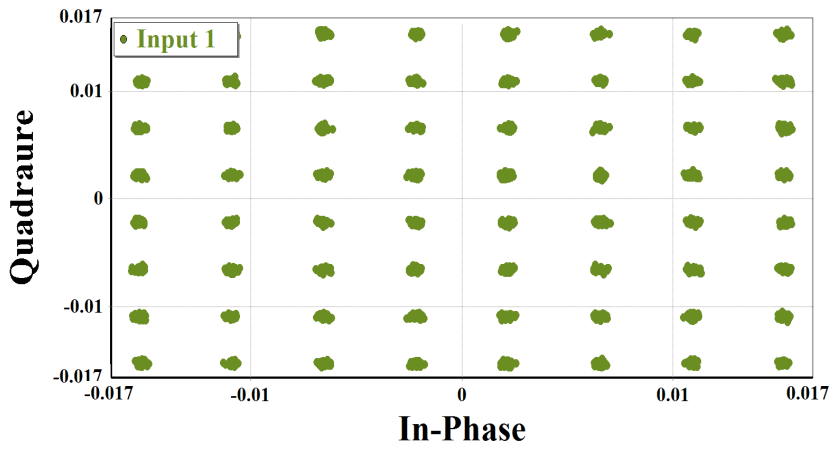

Fig. 9. Constellation diagram of system at full load traffic demand.

\section{CONCLUSION}

In this paper, a two algorithm have been proposed to support traffic exchange between the BBUs and control on the OTXs operation by SS to provide energy saving in the proposed CRAN architecture. Simulation results show that the proposed system can reduce the BBUs utilization by $84 \%$ and $83 \%$ in the residential and office area respectively. The OTXs utilization also reduced by $86 \%$ for 24 hour. The performance of operation of electronic SS has been experimentally studied and proved. To increase the cost effectiveness, the system applied by using the DWDM/SCM-PON-RoF design. In addition, this work introduced a new modification of the power consumption model for C-RAN architecture by adding optical power consumption to the power calculation model.

\section{REFERENCES}

[1] Jaber, M., Imran, M. A., Tafazolli, R., Tukmanov, "5G Backhaul Challenges and Emerging Research Directions: A Survey," A survey. IEEE Access, 4, 1743-1766, 2016.

[2] Zhang, J., Yu, J., Wang, J., Xu, M., Cheng, L., Lu, F., Chang, G. K, "Enabling technologies for millimeter-wave radio-over-fiber systems in next generation heterogeneous mobile access networks," Journal In SPIE OPTO, international Society for Optics and Photonics, pp. 101280A101280A, 2017.
[3] M. Zhu, L. Zhang, J. Wang, L. Cheng, C. Liu and G. Chang, "Radioover-fiber access architecture for integrated broadband wireless services," Journal of Technology, vol. 31, pp. 3614-3620, 2013.

[4] Cheng Liu, Liang Zhang, Ming Zhu, Jing Wang, Lin Cheng and GeeKung Chang, "A Novel Multi-Service Small-Cell Cloud Radio Access Network for Mobile Backhaul and Computing Based on Radio-Over-Fiber Technologies," Journal of Lightwave Technology,vol. 31, pp. 2869-2875, 2013.

[5] R. P. Almeida, R. S. Oliveira, N. S. Moritsuka, C. R. L. Frances, A. Teixeira and J. C. W. A. Costa, "Digital radio over fiber transmission based on SCM and WDM system for C-RAN architecture," in Telecommunications Symposium (ITS), 2014 International, pp. 1-5, 2014.

[6] A. Aloisio, F. Ameli, A. D'Amico, R. Giordano, G. Giovanetti and V. Izzo, "Performance Analysis of a DWDM Optical Transmission System," IEEE Transactions on Nuclear Science, vol. 59, pp. 251-255, 2012.

[7] T. Sigwele et al, "Energy-efficient cloud radio access networks by cloud based workload consolidation for 5G," Journal of Network and Computer Applications, 78, 1-8, 2017.

[8] S. Namba, T. Warabino and S. Kaneko, "BBU-RRH switching schemes for centralized RAN," in Communications and Networking in China (CHINACOM), 2012 7th International ICST Conference on, 2012, pp. 762-766.

[9] B. J. Sahu et al, "Energy-Efficient BBU Allocation for Green C-RAN," IEEE Communications Letters, 2017.

[10] M. Khan, R. Alhumaima and H. Al-Raweshidy, "Reducing energy consumption by dynamic resource allocation in C-RAN," Networks and Communications (EuCNC), 2015 European Conference on, 2015, pp. 169174.

[11] Dalin Zhu and Ming Lei, "Traffic adaptation and energy saving potential of centralized radio access networks with coordinated resource allocation and consolidation," in Communications and Networking in China (CHINACOM), 2013 8th International ICST Conference on 2013, pp. 587-593.

[12] G. Auer, V. Giannini, C. Desset, I. Godor, P. Skillermark, M. Olsson, M. A. Imran, D. Sabella, M. J. Gonzalez, O. Blume and A. Fehske, "How much energy is needed to run a wireless network?," Wireless Communications, IEEE,vol. 18, pp. 40-49, 2011.

[13] C. Lange and A. Gladisch, "On the energy consumption of FTTH access networks," Conference in Optical Fiber Communication , pp. JThA79, 2009

[14] Jayant Baliga, Kerry Hinton and Rodney S. Tucker, "Energy Consumption of the Internet," 32nd Australian Conference on Optical Fibre Technology, COIN-ACOFT 2007.

[15] G. P. Agrawal, "Fiber-Optic Communication Systems," John Wiley and Sons, 2012

[16] Y. Shi, J. Zhang and K. Letaief, "Group sparse beamforming for green Cloud-RAN," IEEE Transactions on Wireless Communications,vol. 13, pp. 2809-2823, 2014.

[17] VPI Photonics, "VPITransmissionMaker Optical Systems," Avaliable in www.vpiphotonics.com/TMOpticalSystems, 2018. 